\title{
Hidden defects in silicon nanowires
}

\author{
M.I. den Hertog, ${ }^{1}$ C. Cayron, ${ }^{2}$ P. Gentile,${ }^{3}$ F. Dhalluin, ${ }^{3,4}$ F. Oehler ${ }^{3}$ T. Baron, ${ }^{4}$ and J.L. Rouviere ${ }^{1}$ \\ ${ }^{1}$ CEA-INAC/UJF-Grenoble1 UMR-E, SP2M, LEMMA, \\ PFNC-Minatec Grenoble, 17 rue des Martyrs, F-38054 \\ ${ }^{2}$ CEA, LITEN, DEHT, LCPEM, Minatec, 17 rue des Martyrs, F-38054 Grenoble Cedex 9, France \\ ${ }^{3}$ CEA-INAC/UJF-Grenoble1 UMR-E, SP2M, SINAPS, \\ 17 rue des Martyrs, F-38054 Grenoble Cedex 9, France \\ ${ }^{4}$ CNRS, LTM, 17 rue des Martyrs, F-38054 Grenoble Cedex 9, France
}

(Dated: October 22, 2011)

\begin{abstract}
Recent publications have reported the presence of hexagonal phases in Si nanowires. Most of these reports were based on 'odd' diffraction patterns and HRTEM images , - 'odd' means that these images and diffraction patterns could not be obtained on perfect silicon crystals in the classical diamond cubic structure. We analyze the origin of these 'odd' patterns and images by studying the case of various $\mathrm{Si}$ nanowires grown using either $\mathrm{Ni}$ or $\mathrm{Au}$ as catalyst in combination with $\mathrm{P}$ or $\mathrm{Al}$ doping. Two models could explain the experimental results : (i) the presence of an hexagonal phase or (ii) the presence of defects that we call 'hidden' defects because they cannot be directly observed on most images. We show that in many cases one direction of observation is not sufficient to distinguish between the two models. Several directions of observations have to be used. Secondly, conventional TEM images i.e. bright field two beam and dark field images, are of great value in the identification of 'hidden' defects. In addition, slices of nanowires perperpendicular to the growth axis can be very useful. In the studied nanowires no hexagonal phase with long range order is found and the 'odd' images and diffraction patterns are mostly due to planar defects causing superposition of different crystal grains. Finally, we show that in Raman experiments the defect rich NWs can give rise to a Raman peak shifted to $504-511 \mathrm{~cm}^{-1}$ with respect to the Si bulk peak at $520 \mathrm{~cm}^{-1}$, indicating that Raman cannot be used to identify an hexagonal phase.
\end{abstract}

PACS numbers:

\section{INTRODUCTION}

Silicon nanowires (SiNWs) are studied widely for use in the semiconductor industry as building blocks for electrical and electro-optical devices. In some semiconductor NW systems such as gallium arsenide ${ }^{1,2}$ both the cubic and hexagonal phase can be observed, which gives rise to interesting physical properties and provides an additional parameter to design functional nanostructures. For SiNWs a controversy is present in literature. In silicon thin films and silicon under high pressure more than twelve forms of silicon have been reported ${ }^{3,4}$, among others the $2 \mathrm{H}$ wurtzite structure. Hexagonal structures were also found in silicon and germanium thin films deposited by laser ablation ${ }^{5,6}$ and recrystallized by an electron beam ${ }^{7}$. The existence of silicon polytypes was deduced using x-ray diffraction ${ }^{8}$, High Resolution Transmission Electron Microscopy (HRTEM) ${ }^{9}$ and Raman studies ${ }^{10}$. In the TEM studies the polytypes appear as a sequence of stacking faults and twin defects, locally ordered on a few nanometers, but no long range order on tens of nanometers was demonstrated. Moreover many articles claim the existence of the $2 \mathrm{H}$ (or $6 \mathrm{H}$ ) hexagonal wurtzite phase in SiNWs ${ }^{11-14}$. The evidence is generally based on transmission electron microscopy (TEM) images in combination with Raman spectra, where a shift from the 520 $\mathrm{cm}^{-1}$ frequency, characteristic of diamond cubic silicon, to lower wavenumbers is observed ${ }^{13}$.

Interestingly very similar TEM images and electron diffraction patterns (EDPs) obtained on thin silicon and gold films and SiNWs have been attributed to double diffraction between superposed twinned crystals ${ }^{15-19}$. Furthermore some of the 'odd' diffraction spots have been attributed to streaking effects caused by very thin defect sequences ${ }^{19,20}$, to which we refer as nanotwins. Such streaking effects can be understood intuitively using the Ewald sphere scheme. A very thin crystal bounded by defects will diffract as a very thin sample with a consequently very long reciprocal lattice rod, that easily intersects with the Ewald sphere and creates a streaking effect. For very thin fcc crystals it has been proposed that at the surface the $\mathrm{ABCABC}$ cubic stacking can be incomplete, therefore some of the crystal can be described by the $2 \mathrm{H}$ wurtzite phase, which can create extra diffraction $\operatorname{spots}^{21}$. This idea was also used for $\mathrm{NWs}^{22}$. Although this hypothesis can explain the experimentally observed EDP's/HRTEM images successfully, this does not exclude the presence of defects.

Interestingly it has also been shown that defects such as dislocations could itself induce the growth of nanostructures, as demonstrated for $\mathrm{PbSe} \mathrm{NWs}^{23}$. In the $\mathrm{Si}$ NWs described in our study no dislocations were observed and therefore we do not expect similar effects.

It seems important to note that the insertion of polytypes with long range order in III-V and II-VI NW systems is remarkably different from most of the claimed observations of polytypes in SiNWs. In III-V and II-VI NWs the different phases are generally separated by grain boundaries perpendicular to the growth direction, what we call here horizontal ${ }^{1,2,24}$. However in SiNWs the polytypes are generally found in the middle of the NW and 
no horizontal boundary is present between the normal diamond cubic structure and the other crystal phase ${ }^{13}$.

In a previous work ${ }^{19}$ we have shown by a comparison of experimental and simulated diffraction patterns that 'odd' diffraction patterns (where odd means that these diffraction patterns do not exist in the perfect diamond cubic crystal) obtained along different directions of observation on SiNWs can be interpreted as a diamond cubic crystal structure with the presence of micro- and nanotwins. This analysis showed that it is not generally possible to determine the crystal phase (hexagonal, cubic or cubic with defects) from one single direction of observation. Despite our warning ${ }^{19}$ recent publications claim to show unambiguous evidence of the existence of an ordered $2 \mathrm{H}$ hexagonal phase and $9 \mathrm{R}$ phase in SiNWs by using correlated TEM with Raman spectroscopy ${ }^{13}$ and electron diffraction ${ }^{14}$, but regarding only one direction of observation in TEM.

The aim of this paper is to complete the work of Ref. 19 (based mostly on EDPs) by describing additional methods to distinguish by TEM the two possible models which are (i) the existence of new phases or (ii) the presence of (twin)defects. The NWs are studied by TEM from several directions and also along the NW growth direction. Focused ion beam (FIB) milling is used to prepare slices of NWs to image defects in the NW section along the growth direction. Furthermore we show that conventional two beam TEM images can be of great value to distinguish between the presence of 'hidden ' defects or random stacking faults oriented along the electron beam direction. Raman spectra were obtained on Ni catalyzed NWs and are compared with literature. Finally, in the discussion part we comment on the TEM images presented in ${ }^{13,14}$ and show that indeed such images can be explained by the presence of micro- and nanotwins in the classic diamond cubic structure.

\section{EXPERIMENTAL DETAILS}

Collaborating with several teams working on Si NW growth, we have observed many nanowires ${ }^{19,25-30}$. In this work we focus on NWs giving unusual HRTEM images or EDPs. We have selected 2 types of nanowires for this paper : (i) Si NWs grown with Ni catalyst and silane as the gas source or (ii) doped Si NWs grown with $\mathrm{Au}$ catalyst. Among, these observed samples, we selected the nanowires that have unusual images or diffraction patterns. It turned out that this happened primarily for two particular growth directions : $\langle 110\rangle$ and $\langle 112\rangle$. The NWs having these growth directions will be respectively called $\langle 110\rangle \mathrm{NWs}$ and $\langle 112\rangle \mathrm{NWs}$. For one particular sample defects could also be observed in other growth direction such as $\langle 111\rangle$ and $\langle 100\rangle$. However, in general $\langle 111\rangle$ NWs, i.e. nanowires with a $\langle 111\rangle$ growth direction, did not have odd images or diffraction patterns.

The NWs were grown by chemical vapor deposition via the vapor-liquid-solid process on a Si substrate in a low pressure chemical vapor deposition (LPCVD) reactor at a base pressure held constant at 20 mbar. Silane $\left(\mathrm{SiH}_{4}\right)$ was the source of Silicon. Hydrogen $\left(\mathrm{H}_{2}\right)$ was used as the carrier gas.

For the Ni catalyst, $2 \mathrm{~nm}$ of nickel was deposited in a vacuum of $10^{-6} \mathrm{~Pa}$ on a cleaned (100) silicon wafer. The substrate was then loaded in the reactor and annealed between 500 and $900^{\circ} \mathrm{C}$ under a $\mathrm{H}_{2}$ flow for several minutes, in order to de-wet the Ni layer and form nanocatalysts. After cooling down the substrate to the growth temperature, $\mathrm{SiH}_{4}$ was introduced by keeping the total pressure $\left(\mathrm{SiH}_{4}\right.$ and $\left.\mathrm{H}_{2}\right)$ constant. Wires were grown at different silane partial pressures ranging from, 0.054 to $0.3 \mathrm{mbar}$ and at temperatures ranging from $550^{\circ} \mathrm{C}$ to $800^{\circ} \mathrm{C}$. Si NWs with different growth directions were obtained. The observed NWs grow primarily in the $\langle 110\rangle$ direction.

Similar conditions were used for the NWs grown with the gold catalyst, except that the NWs were tentatively doped either with $\mathrm{P}$ using diphosphine ${ }^{29}$ or $\mathrm{Al}$ using trimethylalumina ${ }^{25}$ and grown on (111) silicon.

The samples for TEM were prepared in several ways. For the Ni-catalyst samples, the Si substrate with the NWs on top of it was cleaved and the NWs were observed from the side, still attached to the substrate as described in the supporting information of Ref. 29 . A slice of a Ni-catalyst SiNW was prepared by Focussed Ion Beam (FIB) milling with an FEI Strata 400 equipment. Details of the FIB preparation are described in the supporting information. The Au-catalyst samples were either prepared by the cleaving technique or the NWs were deposited on a holey carbon grid. HRTEM images and Electron Diffraction Patterns (EDP's) were realized on a JEOL 4000EX (400 kV), equipped with a GATAN 2Kx2K CCD camera. Scanning TEM (STEM) images were obtained on a FEI TITAN TEM. Raman experiments were realized on an InVia Renishaw spectrometer allowing measurements in the visible and the near InfraRed spectral ranges, equipped with an air-cooled CCD detector, a microscope, and a $50 \times(\mathrm{NA}=0.95)$ objective. Raman spectra were obtained using the $514,5 \mathrm{~nm}$ line of an Argon-Krypton ion laser resulting in a probe size diameter of around $1 \mu \mathrm{m}$.

\section{EXPERIMENTAL RESULTS}

\section{A. $\langle 110\rangle$ Si nanowires with Ni catalyst}

This section is dedicated to Si nanowires grown with $\mathrm{Ni}$ as catalyst. These nanowires have relatively large diameters (in the 100 - $300 \mathrm{~nm}$ range) and grow generally in the $\langle 110\rangle$ direction. Fig. 1 shows typical odd high resolution images and electron diffraction patterns that can be encountered in this sample. The TEM sample was prepared by the clieving technique ${ }^{29}$ and NWs grow mostly in directions not perpendicular to the substrate surface. Therefore the determination of the growth direction of NWs is not so straightforward from a single TEM image, as only a projection of the NW is obtained 
in these images. For instance, in Fig. 1a, the growth direction seems to be $[1 \overline{1} \overline{2}]$, which would be true if the NW was perpendicular to the electron beam, but it was found to be of $\langle 110\rangle$ type by acquiring several HRTEM images taken at different tilt settings and measuring on the CCD camera the angle of the edge of the nanowire. This analysis shows that in Fig. 1 the NW makes an angle of $30^{\circ}$ with the horizontal image plane. The high resolution image of Fig. 1a is composed of two regions. At the edge of the nanowire - region noted Ia in Fig. 1a - the image corresponds simply to a diamond cubic silicon structure observed nearly along a $\langle 110\rangle$ direction. More inside the nanowire - region IIa - the crystal has an additional periodicity of three (1111) planes. The contrast in this region is changing slightly due to thickness variations. Fig. $1 b$ shows the diffraction pattern taken in the microscope. The additional spots due to the new period are clearly visible and will be discussed in the next paragraphs. Then, while keeping the vertical (1111) planes excited, the sample was tilted by approximately $19^{\circ}$ until the diffraction pattern of Fig. 1d was obtained. Along this new direction, high resolution images were taken. One of them is displayed in Fig. 1c. Again this HRTEM image has several regions. At the edge of the nanowire - region Ib - only $\{111\}$ inclined planes can be seen. More inside the nanowire - region IIb - clear spots are obtained. What is surprising is that both the EDP of Fig. 1d and the region IIb of the HRTEM image of Fig. 1c contain 3 families of planes with interplanar spacings close to $0.31 \mathrm{~nm}$ and rotated to each other by of $56^{\circ}$ and $62^{\circ}$ degrees (Fig. 1c-d). Such a direction of observation does not exist in diamond cubic silicon and one could try to build hexagonal structures that display such characteristics.

However, the real nature of the NW appears in conventional TEM (CTEM) images (Fig. 2). Fig. 2a-b are obtained by tilting the sample several degrees off the [110] orientation while keeping one family of $\{111\}$ planes excited. Then an objective aperture is introduced in order to select either the excited $\{111\}$ spot - this is a darkfield- or the transmitted beam -this is the bright field. In the dark field image, regions of different contrast and with triangular shapes can be observed. These regions correspond to different crystallographic grains. A given grain will be bright only if the selected $\{111\}$ spot is in Bragg condition in this grain. On the contrary, a given grain, like the triangular one marked by a $\mathrm{T}$ in Fig. 2a will be dark if the selected $\{111\}$ does not belong to the given grain. If the selected $\{111\}$ spot belongs to different superposed grains, fringes, also known as alpha-fringes ${ }^{31}$ can appear at the interface (like region $F$ in Fig. 2a). Definitely Fig. 2a shows that the NW is not formed of a unique crystal but contains many different grains with different orientations. It should be noted that due to the presence of many grains the deduction of the growth direction can be more complicated than for single crystalline NWs.

To have more details on these defects, a slice of a [011] nanowire perpendicular to the growth direction was realized by FIB on another nanowire. A rather large wire
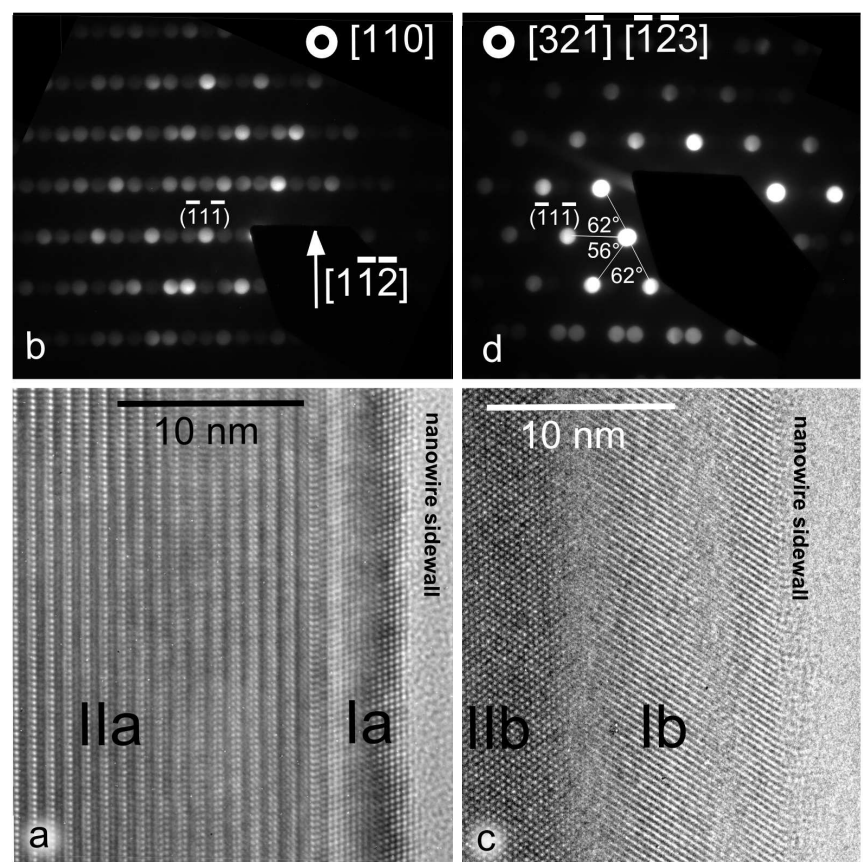

FIG. 1: The same NW on two different ZA's. (a) HRTEM image of a [011] NW observed on the [110] direction. (b) Corresponding diffraction pattern, an extra period of $\sim 1 \mathrm{~nm}$ is present. (c) HRTEM image of the same NW tilted over $19^{\circ}$ - rotating around the (111) plane normal - with respect to the image/EDP in $\mathbf{a}$ and $\mathbf{b}$. (d) The corresponding EDP, the angles are indicated and the distances are equal to the interplanar distance of a $\{111\}$ plane $(0.31 \mathrm{~nm})$.

was selected (diameter of $350 \mathrm{~nm}$ ) in order to facilitate the manipulation of the nanowire after its separation from the substrate. The preparation of the sample is described in the supporting information. Fig. 3 shows several images of the slice. Again, simple conventional images ( Fig. 3a) show that the wire is not uniform but composed of several grains. Fortunately, the slice is quite thin and as evidenced on high resolution STEM images (Fig. 3b,c,d,f), all the observed grains are observed along a [011] direction. Five different types of orientations are found in Fig. 3 hereafter noted A, B, C, D and E. These grains can be related to each others by rotations around the [011] growth direction of the NW (see supporting information for details on transformation matrices) or equivalently by a succession of twining on successive $\{111\}$ planes. Let us choose the orientation named $\mathrm{B}$ in Fig. 3a as our reference crystal. By applying a rotation of angle $\theta=\operatorname{atan}(2 \sqrt{2}) \simeq 70.53^{\circ}($ respectively $-\theta)$ to $\mathrm{B}$, the crystal $\mathrm{C}$ (respectively $\mathrm{A}$ ) is obtained. Grain $\mathrm{C}$ (respectively A) can be deduced from grain B by a twinning on a $(11 \overline{1})$ plane (respectively $(\overline{1} 1 \overline{1})$ plane ) of grain $\mathrm{B}$, which we note $(11 \overline{1}) \mathrm{B}$. Starting from grain $\mathrm{C}$ (respectively A), a rotation of angle $\theta$ (respectively $-\theta$ ) will give grains D (respectively E). The NW is mainly composed of grains $\mathrm{A}$ and $\mathrm{B}$, and twins on $(\overline{1} 1 \overline{1}) \mathrm{B}$ planes but some areas, like areas c,e,f in Fig. 3b nearly contain all the 5 types of grains. Most of the grain boundaries are $\Sigma=3$ GBs located on $\{111\}$ planes. However also $\{112\} \Sigma=3$ 

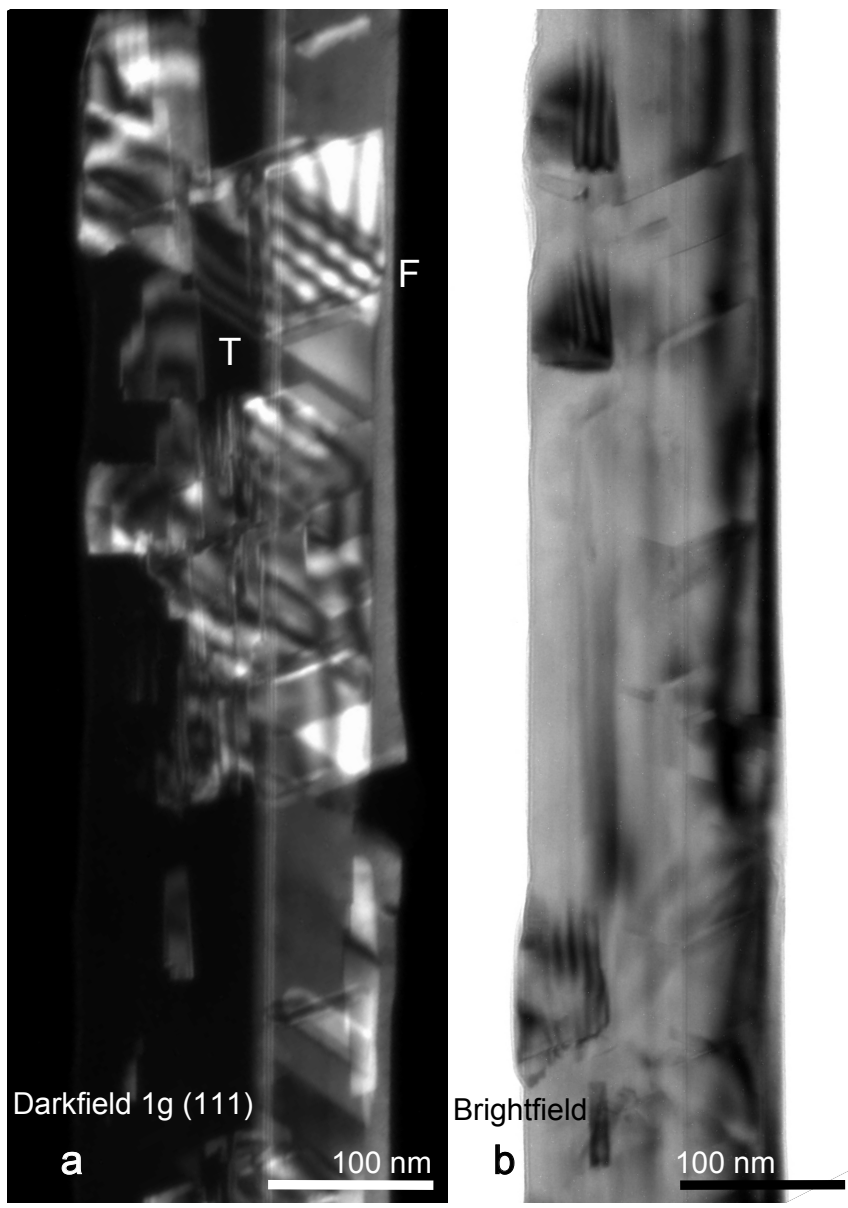

FIG. 2: (a) Darkfield image made using the $1 g$ excitation. The NW was tilted off the [110] ZA by several degrees. (b) Bright field image at the same orientation.

are encountered (Fig. 3d) ${ }^{32}$ and a series of defects on alternating $\{111\}$ and $\{112\}$ planes can lead to an average $\{100\}$ plane (Fig. 3d) in grain B, wich is close to an average $\{110\}$ plane in grain A. As can be seen on Fig. 3b, the FIB milling has increased the thickness of the amorphous layer at the surface of the NW (about $40 \mathrm{~nm}$ of amorphous layer can be seen, where it has a thickness of $2 \mathrm{~nm}$ on traditional images of $\mathrm{NWs}$ ), but it cannot have introduced all the observed defects, because these defects were also present in Fig. 2 and such defects are never observed on FIB prepared specimens of bulk silicon. Such complicated twinning in NWs has also been observed in silver NW's ${ }^{33}$, where a fivefold twin separates the wire in five equal parts.

When the NW is studied from a direction perpendicular or nearly perpendicular to the growth axis, as it is the case in Fig. 1, different crystal grains will superpose in the image/EDP. For instance, let us use the orientations of Fig. 3 to explain Fig. 1b. The diffraction of Fig. 1b can be reproduced by a superposition of twinned grains $\mathrm{A}$ and $\mathrm{B}$ observed along the $[110] \mathrm{B}=[101] \mathrm{A}$ direction whose projection on the (011) plane is marked by a dashed arrow in Fig. 3b. In fact the [110]B direction points out of the plane and makes an angle of $30^{\circ}$ with this dashed

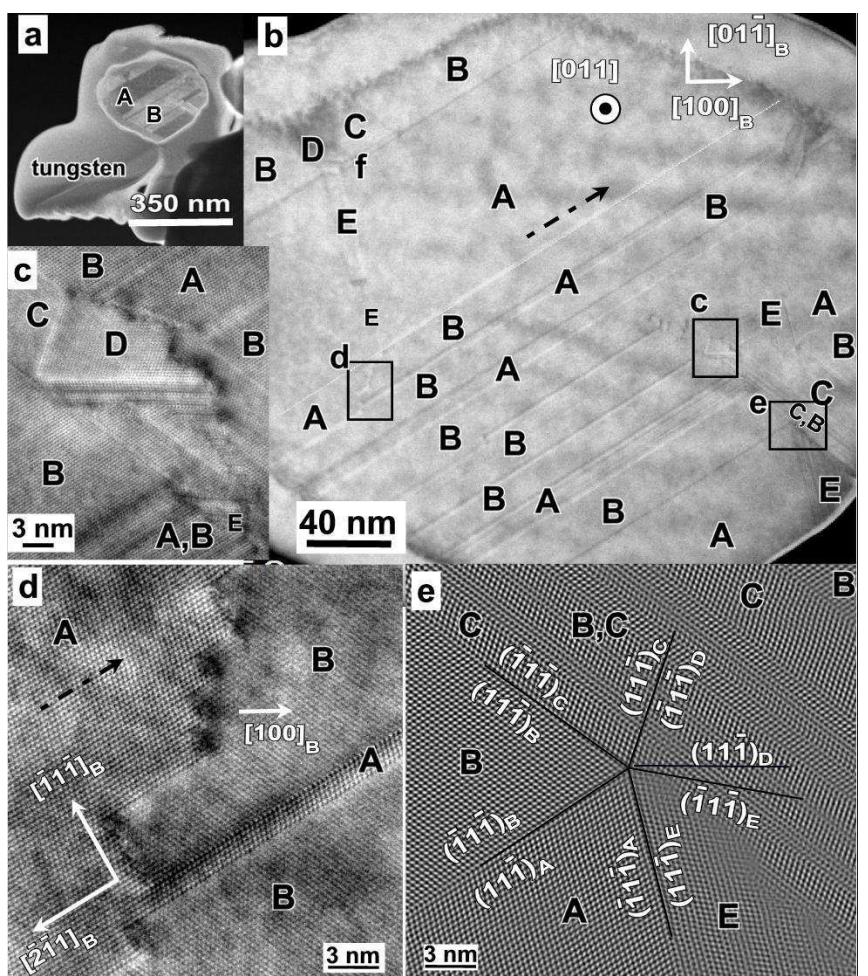

FIG. 3: (a) Bright field (BF) STEM image at low magnification of a NW section, prepared perpendicular to the NW growth direction, observed along the [011] direction. Numerous defects can be observed. Damage due to FIB milling has formed a damaged layer of $\sim 40 \mathrm{~nm}$ around the NW core. (b) BF STEM at higher magnification. (c)BF STEM zoom of a complicated ensemble of defects. Four twin variants can be identified. (d) BF STEM zoom of a series of alternating twin defects on $\{111\}$ and $\{112\}$ planes. (e) (Filtered) HAADF STEM zoom showing 4 different twin variants. The twin grain boundaries are marked by black lines.

arrow. The transformation matrix linking A to B is given in the supporting information as well as a schematic relating the different directions of observations on this type of NW. Fig. 4a is the electron diffraction pattern of the grain $\mathrm{B}$ alone observed along the $[110] \mathrm{B}$, what is called [110]B EDP. In Fig. 4b the [101]A EDP has been simply added to[110]B EDP. In fact, as the two grains are superposed along the electron beam, a beam diffracted by the first grain (let us suppose it is B) can be diffracted again by the second grain (let us suppose that it is grain A). As it is shown in Fig. 4c, many additionnal spots appear due this double diffraction effect. For instance, the spot 1/3(1̄11)A in Fig. 4c can appear with the following mechanism : firstly the incident beam is diffracted by grain $\mathrm{B}$ in the $(1 \overline{1} 3) \mathrm{B}$ direction, which is equal to the $1 / 3(\overline{7} \overline{1} 7) \mathrm{A}$ direction, and secondly this diffracted beam is again diffracted by grain A in the (20) $)$ A direction. The final direction of this double diffracted beam is then $1 / 3(\overline{7} \overline{1} 7) \mathrm{A}+(20 \overline{2}) \mathrm{A}=1 / 3(\overline{1} \overline{1} 1) \mathrm{A}$. In the direct space, the $1 / 3(\overline{1} \overline{1} 1)$ A spot corresponds to a period of 3 times the $\{111\}$ interplanar distance $\left(3 d_{111}\right)$. Fig. $4 d$-e illustrates that this period appears naturally when the two grains $\mathrm{A}$ 
and $\mathrm{B}$ are superposed along the beam. We verified using multislice simulations that the experimentally observed contrast on the $3 d_{111}$ period is well reproduced with the hypothesis of a superposition of two grains along the electron beam, see the supporting information. The contrast in region IIa in (Fig. 1a) would then simply come from the superposition of grain $\mathrm{A}$ and $\mathrm{B}$, the region Ia at the edge of the wire containing only grain A. Having determined a possible structure coherent with Fig. 1a and b, let us try to understand Fig. 1c and d, which are obtained by rotating the nanowire of Fig. 1a along the (1111)B direction with an angle of plus or minus $19^{\circ}$. Grain B observed in Fig. 1a- b in a [110]B direction will be thus observed in Fig. 1c-d along either a [231] or [321] . Let us suppose that this orientation is $[32 \overline{1}]$. Similar results will be obtained with the other possibility. As shown in Fig. 5a grain B oriented along [321] produces an EDP with a systematic row of diffracted spots generated by the (111)B plane. If we found a second grain oriented on a $\langle 321\rangle$ direction whose systematic row generated by its $\{111\}$ planes makes an angle with the (111)B systematic row of $56^{\circ}$, the EDP of Fig. 1d would be explained: the extraspots of Fig. 1d being generated by double-diffraction between the two systematic rows. Using the transformation matrices given in the supporting information we find that when grain B is observed along [321] , the different grains A,C,E,D are observed respectively along directions $[3 \overline{1} 2] \mathrm{A},[\overline{1} 3 \overline{2}] \mathrm{C},[\overline{1} \overline{2} 3] \mathrm{E}$ and $1 / 3[\overline{1} \overline{1} 21] \mathrm{D}$. Grains A, C and $\mathrm{E}$ have the desired direction of observation. Their $\{111\}$

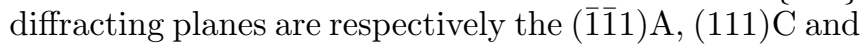
(111) $\mathrm{E}$ and the respective angles of these planes with the (111)B plane are equal to $0^{\circ}$ in grain $\mathrm{A}, 56.25^{\circ}$ in grain $\mathrm{C}$ and $180-56.25^{\circ}$ in grain E. So a superposition of grains B and $\mathrm{C}$ or $\mathrm{B}$ and $\mathrm{E}$ will reproduce exactly the odd diffraction of Fig. 1d. Let us suppose this is grain E. Coming back to Fig. 1c, region Ib contains one $\{111\}$ family of planes that exactly coincides with the (111)E family of planes of Fig. 5c. So region Ib corresponds to a grain E alone, while region IIb corresponds to a superposition of grains $\mathrm{B}$ and $\mathrm{E}$ along the electron beam. So while tilting the sample from the orientation of Fig. 1a to the orientation of Fig. 1b, the studied area of the nanowire was changed slightly without notice. In Fig. 1a this is an area with a superposition of grain A and B at the center with a grain A at the edge, while in Fig. 1b there is a superposition of grain $\mathrm{B}$ and $\mathrm{E}$ at the center with a grain $\mathrm{E}$ at the edge. This is not very surprising as (i) the nanowire contains many grains as can be seen in Fig. 2 and as TEM goniometers are not all eucentric and always produce a shift when tilting. All this analysis shows that a superposition of grains is at the origin of the experimental data and it is not necessary to introduce exotic crystal phases to explain them.

Raman spectra were obtained on these NWs. The majority of the studied wires showed a Raman spectrum similar to that of bulk diamond cubic silicon which has a peak at $520 \mathrm{~cm}^{-1}$. However, as show in Fig. 6, some NWs analyzed in the present study showed a shift of the Raman peak to lower wavenumbers. In these cases the spectra obtained on the extremities and mid-
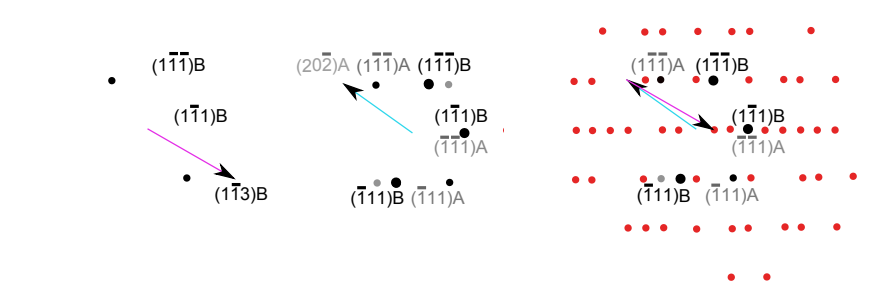

a grain B: ZA [110]B

b grain B: ZA [110]B

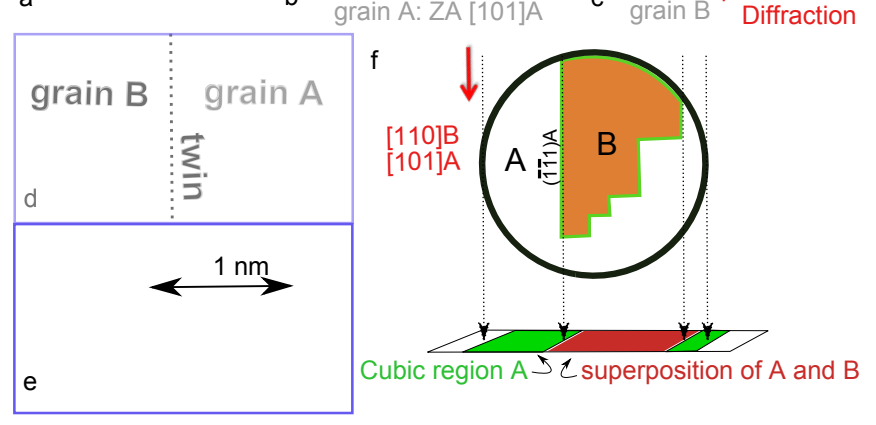

FIG. 4: (a) Electron diffraction of grain B along the [110]B direction. (b)Combined [110]B EDP and [101]A EDP, grain A and $\mathrm{B}$ are twin crystals, the twin plane being $(11 \overline{1}) \mathrm{A}=(\overline{1} 1 \overline{1}) \mathrm{B}$. (c) EDP when the electron beam crosses first grain B then grain A. Double diffraction creates additional spots, in particular the $1 / 3\{111\}$ spot. (d) Atomic model of a 111 twin grain boundary between grain A and B. Grain B is on the left and grain A on the right of the grain boundary, as depicted on the scheme. (e) Projection of a superposition of grains A and $\mathrm{B}$ along the electron beam. The period of 3 times the $\{111\}$ interplanar distance associated to the $1 / 3\{111\}$ spatial frequency appears. (f) Schematic illustrating a possible configuration of grain A and B in the NW volume and how these grains superpose in the TEM image, based on Fig. 3d.

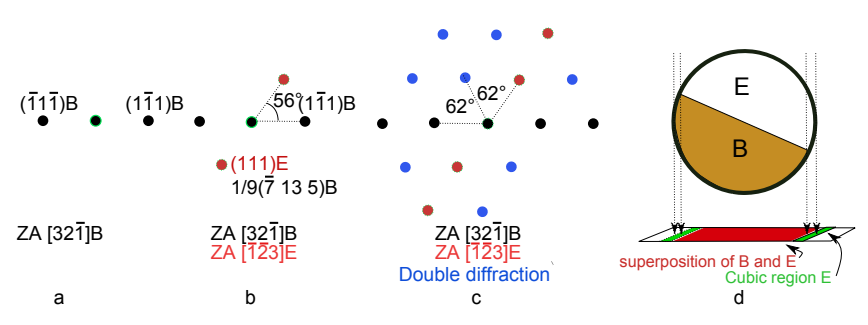

FIG. 5: (a) The EDP of crystal B observed along a [32̄1] direction contains only a systematic row of diffraction spots corresponding to a $\{111\}$ plane (b) The EDP of crystal E ob-

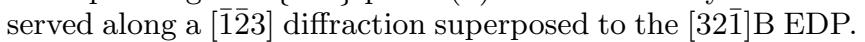
The angle between the two systematic rows of the two grains is $56^{\circ}$. (c) Similar to Fig. 4c: when grains B and E are superposed along the electron beam, additional spots appear in the EDP due to double diffraction (blue).(d) Schematic illustrating a possible configuration of grain B and E in the NW volume and how these grains superpose in the TEM image, based on Fig. 3e..

dle of the NW were different (Fig. 6). At one end the vibrational mode was relatively broad and observed around $484 \mathrm{~cm}^{-1}$, which could be attributed to amorphous silicon $^{34}$. In the middle a peak at $504 \mathrm{~cm}^{-1}$ was observed, whereas the other wire extremity showed a peak close to the value of diamond cubic silicon $\left(519 \mathrm{~cm}^{-1}\right)$. 


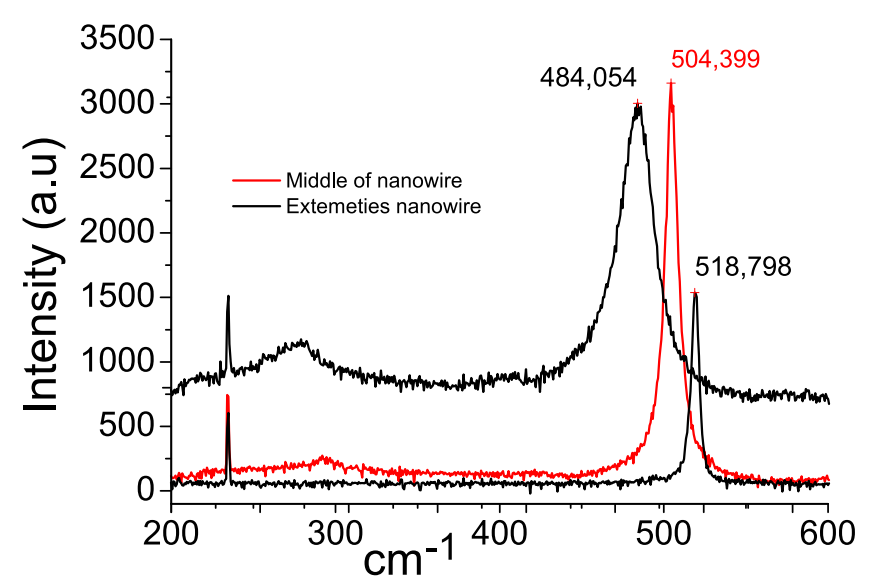

FIG. 6: Example of three Raman spectra made on the same NW in the middle and on both ends of the NW.

In literature calculations can be found showing that the Raman spectrum of the silicon wurtzite structure contains a peak around $503-506 \mathrm{~cm}^{-110,35,36}$. In other experimental papers ${ }^{37,38}$ Raman peaks at either 504$511 \mathrm{~cm}^{-1}$ or $517 \mathrm{~cm}^{-1}$ are observed. They are attributed to the presence of defects that create smaller grains inside the NW. Indeed, due to quantum confinement, the Raman frequency should be downshifted for decreasing grain sizes

Since all TEM evidence presented in the foregoing unambiguously shows that no other crystal structure than diamond cubic silicon is present, the shifted Raman signal is attributed to the high twin density, as the defects can create locally $2 \mathrm{H}$ ordering on the range of a few atomic planes ${ }^{39}$ and as this local ordering can be seen in some areas of Fig. 3c and e. Intuitively it seems that a varying defect density from the perfect wurtzite phase on one side and the diamond cubic phase on the other side can tune the Raman signal everywhere between 503 and $520 \mathrm{~cm}^{-1}$. Also a decreasing crystal grain size will increasingly downshift the Raman resonance frequency ${ }^{38}$.

\section{B. Doped Si nanowires with Au catalyst}

In the case of gold catalyzed NWs (grown in the presence of TMA or phosphine) that grow mostly in the $\langle 112\rangle$ direction, we observed two different cases. Either images similar to Fig. 1a where the $3 d_{111}$ period is very regular, were observed (see supporting information Fig. 11). Or images like Fig. 9 were observed. The question is again to determine if the observed images are due to a superposition of grains or to new phases. Each case is studied in turn.

\section{Doped Si nanowires grown with TMA}

Gold catalyzed NWs grown in the presence of TMA were observed to grow in various growth directions being $\langle 112\rangle,\langle 111\rangle$ or $\langle 100\rangle$ and would often change growth direction along the NW. In the supporting information (Fig. 11a ) a detail of a $\langle 112\rangle$ NW having a $3 d_{111}$ period in its center is shown. In Fig. 7 a detail of a $\langle 111\rangle$ NW is shown. Three regions of the NW separated by (111) planes can be distinguished. They are marked as I, II and III. Region I has the normal diamond cubic structure. Region II shows the $3 d_{111}$ period everywhere. In region III the $3 d_{111}$ period disappears in the middle of the image without the observation of a grain boundary and the left side of region III has again the normal diamond cubic structure. As for the $\langle 011\rangle$ NW and as schematically shown in Fig. 4, it is clear that in this NW, the $3 d_{111}$ period can be explained by a superposition of two crystal grains along the electron beam that are in a twin relation, which is supported by the smooth disappearance of the $3 d_{111}$ period in region III of Fig. 7 that is attributed to an inclined defect that reaches the NW surface. This smooth disappearance would be more difficult to explain if a new phase was present in the center of the wire and occupied the whole thickness of the nanowire. The easiest way to distinguish between these two possibilities (superposition of grains or new phase) is to acquire a conventional image as shown in Fig. 8b obtained on a different NW. This image is obtained using the common $\{111\}$ beam having tilted the sample $11^{\circ}$ off the ZA. One can notice bright fringes in the center of the wire which have no relation in number to the $3 d_{111}$ periods observed in the HRTEM image of Fig. 8a obtained at the same region of the NW. In fact, in litterature, such fringes are known as alpha fringe contrast and come from the interferences between the two superposed grains ${ }^{31}$. Alpha fringes are due to grain boundaries inclined to the electron beam that result in a varying thickness of both crystal grains. The interference of beams passing through both grains create fringes comparable to thickness fringes. The periodicity of alpha fringes depends on many parameters and is therefore difficult to analyze, but we can distiguish two different situations (i) the periodicity of the fringes is equal to the number of $3 d_{111}$ periods indicating a regular repetition of a grain $\mathrm{A}$ and grain $\mathrm{B}$ with grain boundaries parallel to the electron beam extending through the entire NW volume where only one of two grains is in a difracting condition. Such a regular repetition can be viewed as an anomalous crystal phase other than the diamond cubic structure and will be discussed in the next section. (ii) the periodicity of the fringes is not equal to the number of $3 d_{111}$ periods (as is the case in Fig. 8) and must therefore be due to alpha fringe contrast caused by the superposition of two crystal grains both in a diffracting condition

\section{Doped $\langle 112\rangle$ Si nanowires grown with phosphine}

Fig. 9 shows the case of gold catalyzed NWs grown in the presence of phosphine ${ }^{29}$ and growing in the $\langle 112\rangle$ direction. These nanowires were grown on a $\langle 111\rangle$ Si substrate. Most of the nanowires had a $\langle 111\rangle$ growth direction and these $\langle 112\rangle$ NWs were in fact in minority. Most 


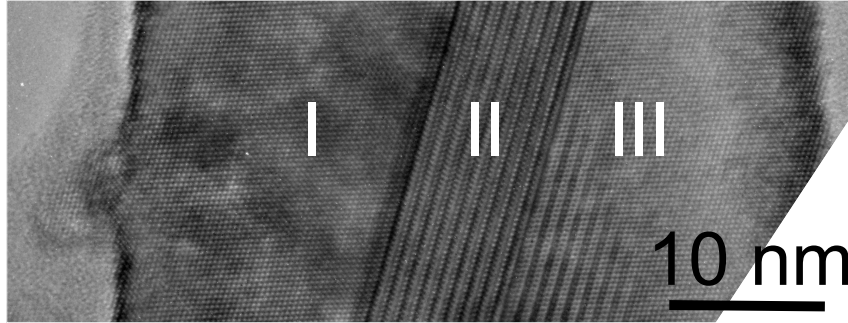

FIG. 7: HRTEM detail of a NW made on the [011]ZA that contains three regions I (cubic structure), II ( $3 d_{111}$ period) and III (smooth disapearance of $3 d_{111}$ period and again cubic structure).

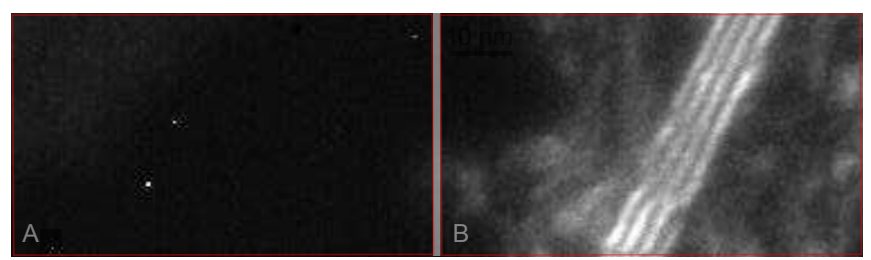

FIG. 8: (a) HRTEM detail of a NW that contains six $3 d_{111}$ periods made on the [011]ZA. (b) Conventional image of the same region made using a (111) reflection showing four bright fringes.

of the observed $\langle 112\rangle$ NWs had variations of contrast on $\{111\}$ planes parallel to the growth direction, that can be seen in Fig. 9. On the HRTEM image of Fig. 9a the NW is observed along a [011] direction and has a diameter of $66 \mathrm{~nm}$. Clearly at the left edge of the NW there are two grains, $\mathrm{A}$ and $\mathrm{B}$, that are in a twin relationship and that occupy the whole thickness of the NW as the contrast of the HRTEM image is sharp. As the electron beam is not perfectly along the common [011] direction but a few degrees off, the A and B grains are not observed exactly in the same direction and grains in the A orientation are darker than the ones in the B orientations. In the center of the NW there are a series of dark lines on (111)B planes. The first three dark lines on the left are regularly separated by a distance equal to $3 d_{111}$. Then there is another group of two lines separated again by $3 d_{111}$. The question is whether these $3 d_{111}$ regions (like the one outlined by a white double arrow) are due to a local ordering of stacking faults (see Fig. 9e) or whether they are due to a superposition of two grains along the electron beam (like in Fig. 4c). The answer is again given by conventional TEM images. Fig. $9 \mathrm{~b}$ is a conventional TEM image obtained by selecting the (1̄11) plane of crystal A that does not correspond to a $\{111\}$ plane in B. If there were a superposition of two grains (as shown in Fig. 4f) the contrast on the 3 first periods (delimited by an arrow in Fig. 9a) would be uniform as this area would be composed of a constant amount of crystal A, however we clearly see several regions and the number of bright lines in Fig. 9b corresponds to the number and location of $3 d_{111}$ periods in Fig. 9a (for example compare the regions oulined by the arrow) which is exactly the situation as described by (i) in the previous section. So definitely, contrary to Fig. 8, we have a succession of stacking faults crossing the whole thickness of the nanowire. The exact stacking order is determined by analyzing HRTEM images and by looking at the alignment of the white dots along the $[\overline{2} 1 \overline{1}]$ B direction (Fig. 9d). When the tunnels of the [011] direction of observation are white (as in Fig. 9d), the cubic stacking abc of orientation B is kept as long as the tunnels are aligned along $[\overline{2} 1 \overline{1}] \mathrm{B}$. When a shift in the dot alignment takes place, it is the inverse stacking cba of orientation A that occurs and locally there is a small portion of crystal A around the stacking fault (see Fig. 9d). For instance, following the line in Fig. 9d, we have the following stacking : abc-bca-cab-abc ie 3 staking faults regularly distributed every $3 d_{111}$ period. On the right of these regularly distributed stacking faults, there is a series of random stacking faults.

\section{DISCUSSION}

Having observed a selection of nanowires having odd images/diffraction patterns, we have found two different situations. In undoped $\langle 110\rangle$ NWs grown with a Ni catalyst and $\mathrm{Al}$ doped NWs grown with Au catalyst, the odd images/EDPs are definitely due to a superposition of twins along the electron beam. So in this first case, the odd situations are due to (mostly $\{111\}$ ) planar defects our 'hidden' defects that create superposition of twinned crystals. In P doped $\langle 112\rangle$ NWs grown with Au catalyst, we observed a more or less regular distribution of SFs that cross the whole diameter of the nanowire. In this second case, there is no superposition of twins or defects along the electron beam. Of course, if the stacking faults would be regularly distributed there would be a creation of hexagonal phases as claimed in ${ }^{11,13}$, which would be $2 \mathrm{H}$ if the stacking faults are every $2 d_{111}$ planes or $9 \mathrm{R}$ if the stacking faults are distributed every $3 d_{111}$ planes. However, in all our observed samples, the stacking faults were never distributed regularly on large distances and therefore it seems misleading to describe the Si nanowires as having an hexagonal phase. Interestingly these vertical SFs on $\langle 112\rangle \mathrm{NWs}$ and inclined defects are frequently observed when NWs are tentatively doped. So it seems likely that dopants or impurities are at the origin of the observed defects and might segregate at these defects. These faulted nanowires could be a way to incorporate dopants or impurities and could have interesting properties if the defects were more controlled. In any case more experimental data will be necessary to prove that such stacking faults are really due to impurities.

So given the respective TEM and Raman characterizations performed on $\mathrm{Ni}$ and $\mathrm{Au}$ catalyzed silicon NWs it seems that no hexagonal phase with long range order is present in the studied NWs that grow in majority in the $\langle 112\rangle$ or $\langle 110\rangle$ direction. In all our observed cases the appearance of a regular period of $3 d_{111}$ is due to a superposition of different crystal grains caused by inclined (hidden) defects. Some clues can indicate when this situation is likely to happen : (i) the anomalous crystal phase 


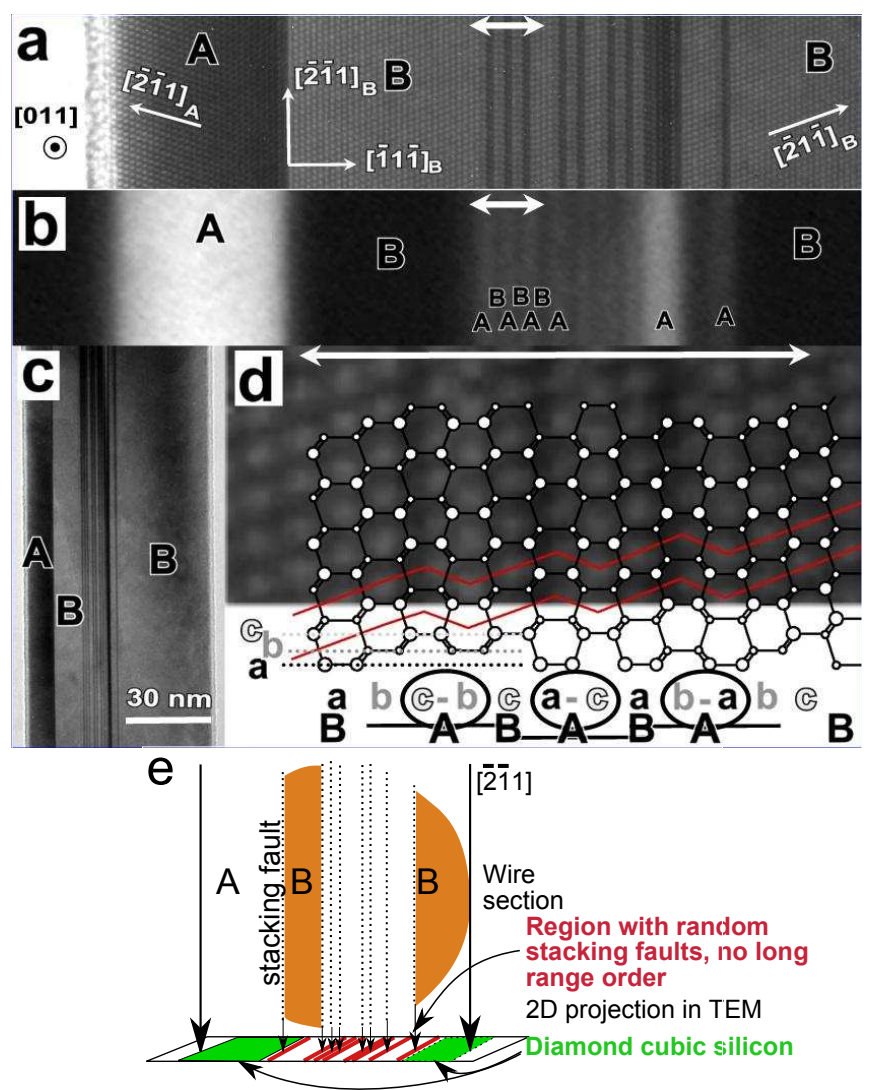

FIG. 9: (a) HRTEM image of a part of gold catalyzed $\langle 112\rangle$ NW observed along the [011] direction. Atoms are black. Two crystal orientations, marked as $\mathrm{A}$ and $\mathrm{B}$, in a twin relationship are present. The vertical twin plane is chosen as $(\overline{1} 1 \overline{1}) \mathrm{B}$ $=(11 \overline{1}) \mathrm{A}$. The length of the white double arrowed line is 10 $d_{111} \sim 3.14 \mathrm{~nm}$ (b) Dark field image of the same nanowire by tilting the sample away from the [011] direction in order to be on a two-beam condition with crystal A (beam excited $\mathrm{g}=(\overline{1} 1 \overline{1}) \mathrm{A})$ and to have no excited beam on crystal B. (c) Bright field general view of the nanowire on the [011]. (d) HRTEM image zooming on the area in a outlined by the double white arrow. An atomic model of the projected structure is superimposed on the experimental images. The stacking sequence of $(\overline{1} 1 \overline{1}) \mathrm{B}$ planes is marked by the letter a-b-c and outlined by lines joining the center of the tunnels of the structure. The order a-b-c is characteristic of the orientation $\mathrm{B}$, while the reverse order c-b-a, outlined by ellipses around the letter c-b-a, is characteristic of the orientation A. Stacking faults make the boundaries between grain A and B. Alternatively, one can consider that there are locally some $2 \mathrm{H}$ hexagonal phases b-c-b-c or c-a-c-a or a-b-a-b in the orientation B. (e)Schematic of a NW section on the $[\overline{2} \overline{1} 1] \mathrm{ZA}$, which is the growth direction. The projection of the stacking faults in the TEM image is illustrated.

is only observed in the center of the NW, the thicker region, and not at the edge of the NW where the crystal is thin, which makes it very probable that this odd contrast is caused by superposition of different crystal grains (e.g. Fig. 1), (ii) twins are often found in the vicinity of the odd region and (iii) the odd contrast can disappear smoothly in the middle of the NW, without the presence of a visible grain boundary or defect (e.g. Fig. 7). In the studied NW systems 'hidden' defects were evidenced either by a FIB slice of the NW or more easily and generally by taking conventional TEM images of the NWs. 'hidden' twins are then revealed by inhomogeneous contrast in dark-field and two beam images (Fig. 2a-b) or alpha-fringe contrast (Fig. 2a and Fig. 8b). One should not use only HRTEM images associated with only one diffraction pattern - as it is often the case - to claim the existence of hexagonal phases. We have seen in Fig. 1 that two HRTEM images of the same NW obtained in two directions can also be misleading if the NW contains different regions with different structures.

Of course, recent improvements in TEM techniques should allow other ways to characterize the NWs. For instance, one could eventually use the STEM sectioning technique $^{40}$ to visualize the individual grains. By using a large convergent beam and a probe-Cs-corrector it was shown that when the probe is focused at the top surface of the sample, essentially the top grain is visualized. When the probe is focused at the bottom surface of the sample, a superposition of the top and bottom grain was obtained ${ }^{41}$. Another solution would be to realize a $3 \mathrm{D}$-analysis of grains like in Ref. 42. However these experiments are not routine analysis yet. On the contrary qualitative CTEM images can be realized quickly and efficiently on any kind of TEM.

Papers claiming the observation of the hexagonal structure in Si NWs often use the shifted Raman frequency to $503-506 \mathrm{~cm}^{-1}$ as evidence in combination with TEM images, where generally only one direction of observation is regarded ${ }^{13}$. A recent $\operatorname{article}^{43}$ reports odd HRTEM images on SiNWs in combination with a shifted Raman signal to $503 \mathrm{~cm}^{-1}$, however only normal diamond cubic silicon is observed in X-ray diffraction (XRD) experiments (see ref 43 and the supporting information). This is a clear indication that Raman spectroscopy is not the appropriate technique to distinguish between the hexagonal phase and the diamond cubic phase with defects because a shifted Raman signal does not necessarily imply the presence of $2 \mathrm{H}$ or $9 \mathrm{R}$ phases ${ }^{38,43}$. From our point of view Raman spectra with only one associated HRTEM image cannot be a proof of the presence of hexagonal phases.

We do not refute the existence of a $2 \mathrm{H}$ structure in all the Si NWs. Such structures can exist but are in general localized to a very limited area and are constituted by a limited number of stacking faults, as shown in Ref. 38. Most publications claiming the existence of a $2 \mathrm{H}$ structure do not bring sufficient evidence and the results can be interpreted by a classical Si structure with nano and microtwins ${ }^{19}$. A very convincing example of $2 \mathrm{H}$ hexagonal silicon is the growth of a silicon shell on a $\mathrm{GaP} \mathrm{NW}$ with the $2 \mathrm{H}$ hexagonal structure ${ }^{44}$, where it is shown that the crystal structure of the core GaP $\mathrm{NW}$ that has the $2 \mathrm{H}$ hexagonal structure can be successfully transferred to the Si shell. In this case none of the 'odd' features as described previously (i - iii) can be observed. The presence of massive $2 \mathrm{H}$ structures in pure $\mathrm{Si}$ NWs inserted perpendicular to the NW axis (as in the GaAs $\mathrm{NWs}^{1,2}$ ) between diamond cubic silicon regions is 
more convincingly reported by Arbiol et al. ${ }^{45}$ in the very special case of Si NWs elaborated at high temperature $\left(>700^{\circ} \mathrm{C}\right)$. However, the FFT of their Figure $4^{45}$ shows odd features (the distance $\mathrm{X} 1-\mathrm{X} 2 \neq \mathrm{X} 2-\mathrm{X} 3$ ). Therefore conclusive evidence whether an hexagonal phase or a cubic phase with defects is present can only be obtained by using several directions of observation in TEM, especially using conventional TEM images in association with HRTEM/EDP images, or by correlating TEM and XRD measurements.

\section{CONCLUSION}

In conclusion studying several cases of Si NWs presenting odd images or diffraction patterns, we do not observe massive hexagonal phases extending over tens of $\mathrm{nm}$. In some situations of doped $\langle 112\rangle \mathrm{NWs}$, there is a tendency to form more or less regular vertical stacking faults. But from our point of view it seems misleading to consider this local ordering as hexagonal Si phases. In most cases, odd diffraction patterns/ TEM images are due to a superposition of different crystal grains along the electron beam. Generally these grains are in a twin relationship and form what we call the 'hidden' defects that are at the origin of the odd contrasts. The easiest way to check whether there is a superposition or not of grains along the electron beam is to perform conventional TEM images. In the study of $\langle 110\rangle$ NWs, a slice of the NW, perpendicular to the growth direction and realized by FIB, was very helpful to understand the different grains present in the NWs. Finally we show that defect rich NWs can give rise to shifted Raman peaks, which can be attributed to the small crystal grain size created by the high defect density $^{38}$.

\section{ACKNOWLEDGEMENTS}

The authors thank the European Community for funding under the FP6 Marie Curie Host Fellowships for Early Stage Research Training (EST) "CHEMTRONICS" contract No. MEST-CT-2005-020513. We would like to acknowledge V. Salvador (CEA-Grenoble - INAC) for the skilful FIB sample preparation. We thank Eddy RomainLatu (CEA-Grenoble - DOPT - LETI), Denis Rouchon (INPG, LEPMI - LETI) and Michel Mermoux (LEPMI, CNRS-INPG-UJF) for help with the Raman measurements. We thank Heinz Schmid, Mikael T. Björk, Heike Riel, Siegfried Karg and Walter Riess (IBM Zurich) for one NW sample.

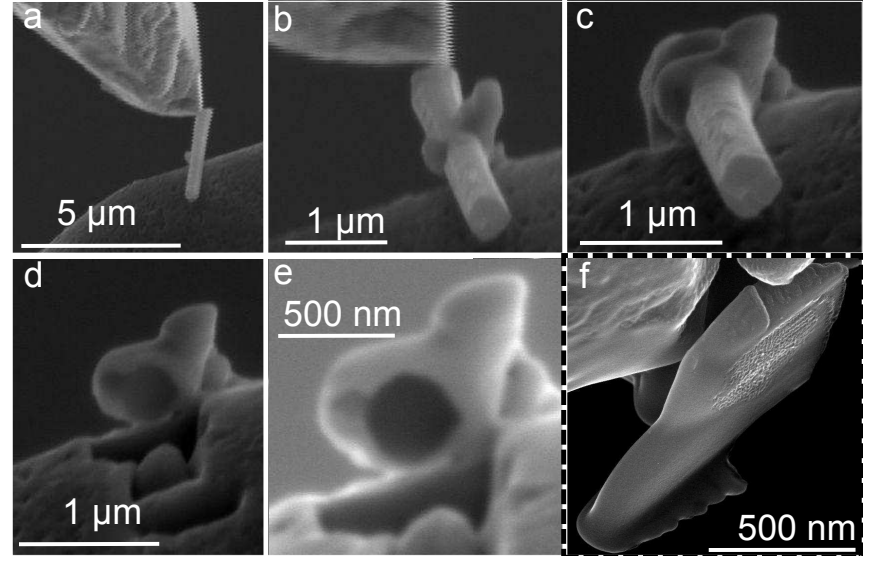

FIG. 10: SEM images obtained during FIB preparation steps preparing a slice of NW that can be observed along the growth direction. (a) The NW attached with W to the W tip, the omniprobe, is approached to the TEM grid. (b) The NW is attached using $\mathrm{W}$ deposition to the TEM grid. (c) The bottom part of the NW is gradually removed by FIB milling using progressively decreasing acceleration voltage to minimize amorphization of the specimen surface. (d) The upper part of the NW is removed by FIB milling as in (c). (e) The sample is finally thinned to electron transparency. (f) After TEM observation a gentle 20s Argon ion milling was attempted to remove some of the amorphous surface layers. Unfortunately this has broken the sample.

\section{SUPPORTING INFORMATION}

\section{A. FIB sample preparation}

For FIB sample preparation the NW was attached to the omniprobe tip using W. Then the NW was removed from the substrate by FIB milling at the NW base. The NW was approached to the copper TEM grid (Fig. 10a) and attached to the TEM grid by W deposition(Fig. 10b). The NW was gradually thinned to electron transparency by using progressively decreasing acceleration voltages to minimize the thickness of the amorphized surface regions on the TEM sample (Fig. 10c-e). After TEM observation of the NW slice as shown in Fig. 3 a gentle 20s Argon ion milling was attempted to remove some of the amorphous surface layers. Unfortunately this has broken the sample (Fig. 10f). The SEM image Fig. 10f shows that the thickness of the slice can be estimated around $130 \mathrm{~nm}$. The FIB milling/ embedding has partly damaged some surface of the nanowires, but it cannot have introduced all the observed defects, because these defects were also present in Fig. 2.

\section{B. Multislice simulation}

In Fig. 11a-b two TEM images of a region with odd contrast, similar to the one in Fig. 1a and Fig. 7, are shown and compared to JEMS multislice simulations ${ }^{46}$ at Scherzer defocus. In the simulation the grains $\mathrm{A}$ and 


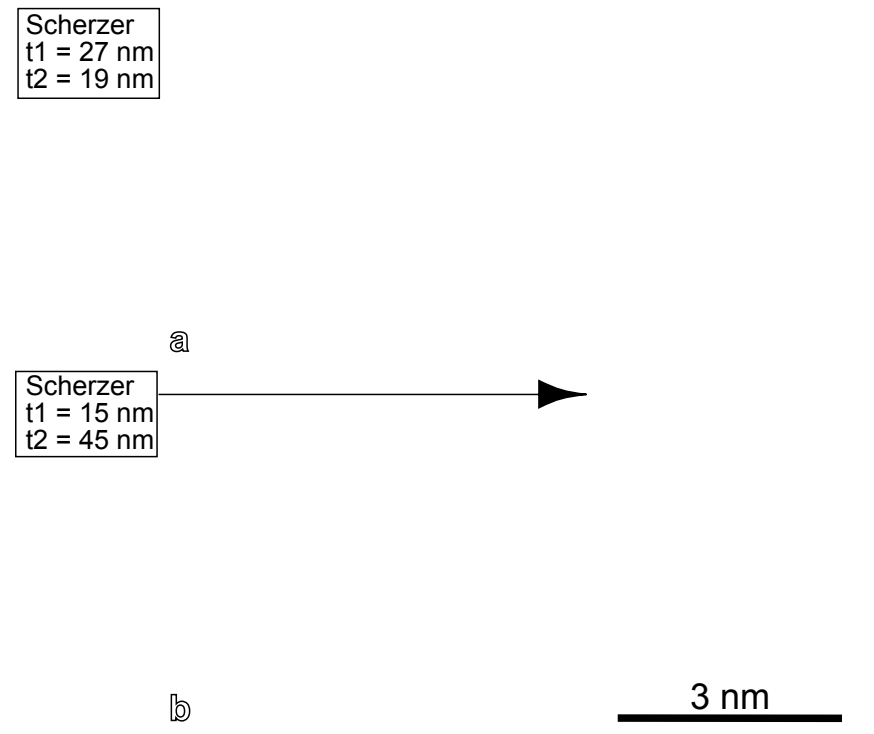

FIG. 11: (a-b) HRTEM images of a defect zone on the [110] ZA. The best match with multislice simulations of two superposed crystal grains related by a twin defect placed on a $\{110\}$ plane is shown in the inset at Scherzer defocus. The thickness of both crystal grains, indicated by $t 1$ and $t 2$ is indicated. The characteristics of the JEOL $4000 \mathrm{EX}$ were included in the simulations.

B were simply superposed along the direction of observation with respective thicknesses $t_{A}$ and $t_{B}$. The complete crystal thickness was around $50 \mathrm{~nm}$, corresponding to the NW diameter. The electron plane wave first propagated in grain B then in the twinned grain A. The experimentally observed contrast and the $3 d_{111}$ period are well reproduced in the simulations.

\section{Transformation matrices}

In this appendix, the transformation matrices that link the different grains A, B, C, D, and E as described in Fig. 3 are given. $\mathrm{B}$ is taken as the reference crystal. Let $\mathrm{X}$ be a vector whose coordinates in crystal $\mathrm{B}$ (respectively $\mathrm{A}$ ) are given by the column vector $X_{B}$ (respectively $X_{A}$ ). The transformation matrix from grain B to grain A (noted B_to_A) links the two column vectors: $X_{A}=$ B_to_A $* X_{B}$. It is a rotation matrix around the $[011] \mathrm{A}=[011] \mathrm{B}$ axis with an angle of about $-70.53^{\circ}$ (see Fig. 3). For instance [111̄]]B (respectively [110]B) has coordinates $[11 \overline{1}] \mathrm{A}$ in grain A (respectively [101]A)

$$
\begin{array}{ll}
\text { A_to_B }=\frac{1}{3}\left(\begin{array}{rrr}
1 & -2 & 2 \\
2 & 2 & 1 \\
-2 & 1 & 2
\end{array}\right) & \text { B_to_A }=\frac{1}{3}\left(\begin{array}{rrr}
1 & 2 & -2 \\
-2 & 2 & 1 \\
2 & 1 & 2
\end{array}\right) \\
\text { B_to_E }=\frac{1}{9}\left(\begin{array}{rrr}
-7 & 4 & -4 \\
-4 & 1 & 8 \\
4 & 8 & 1
\end{array}\right) & \text { B_to_D }=\frac{1}{9}\left(\begin{array}{rrr}
-7 & -4 & 4 \\
4 & 1 & 8 \\
-4 & 8 & 1
\end{array}\right)
\end{array}
$$

TEM the direction of observation changes differently for the various grains. This effect is illustrated schematically in Fig. 12 for grains A and B of Fig. 3 where stereograms of grain A and B are shown if the NW is observed along the growth direction (as in Fig. 3) and if the NW is observed from the side along $\{110\}$ and $\{123\}$ type directions respectively (as in Fig. 1). Such a schematic is helpful for better understanding of the observation of the different grains and correct indexing of diffraction patterns obtained on superposed grains.

When a NW containing multiple grains is tilted in the 


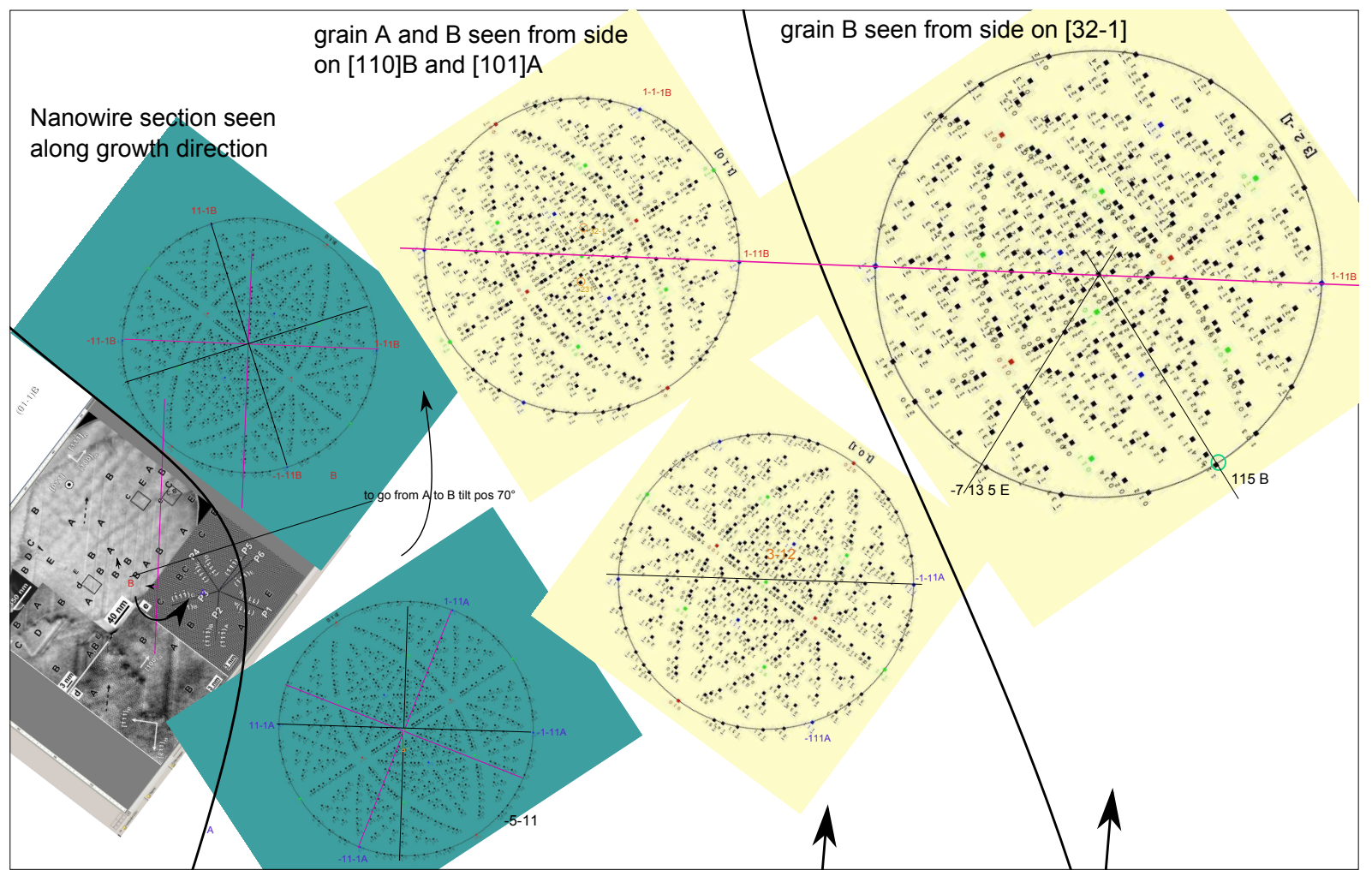

FIG. 12: Schematic illustrating the different directions of observation of grain A and B observed in Fig. 3 with stereograms. Most to the left grain A and B are observed along the growth direction as in Fig. 3, in the center grain A and B are observed along a $\{110\}$ type direction. On the right grain $\mathrm{A}$ and $\mathrm{B}$ are observed along a $\{123\}$ type direction. The arrows in the center of the stereogram indicate how the NW is positioned in the image plane (as pointed out previously the NW is not lying flat in the image plane and the electron beam is not perpendicular to the NW). The arrows below the stereogram indicate how the projection of the electron beam enters the NW slice and a small copy of Fig. $3 \mathrm{~b}$ and e is repeated here for clarity. The schematics of Fig. 4f and Fig. 5d are based on how the different grains would superpos in the respective directions of observations if the electron beam would enter the NW slice as indicated.

${ }^{1}$ F. Glas, J. Harmand, and G. Patriarche, Phys. Rev. Lett. 99, 146101 (2007).

${ }^{2}$ F. Davidson, D. Lee, D. Fanfair, and B. Korgel, J. of Phys. Chem. C 111, 2929 (2007).

${ }^{3}$ R. Wentorf and J. Kasper, Science 139, 338 (1963).

4 J. Besson, E. Mokhtari, J. Gonzalez, and G. Weill, Phys. Rev. Lett. 59, 473 (1987).

${ }^{5}$ Y. Zhang, Z. Iqbal, S. Vijayalakshmi, and H. Grebel, Appl. Phys. Lett. 75, 2758 (1999).

${ }^{6}$ Y. Zhang, Z. Iqbal, S. Vijayalakshmi, S. Qadri, and H. Grebel, Solid Sate Comm. 115, 657 (2000).

7 J. Parsons and C. Hoelke, Phil. Mag. A 50, 329 (1984).

${ }^{8}$ M. Hendriks, S. Radelaar, A. Beers, and J. Bloem, Thin
Solid Films 113, 59 (1984).

${ }^{9}$ U. Dahmen, C. Hetherington, P. Pirouz, and K. Westmacott, Scripta Metallurgica 23, 269 (1989).

10 J. Bandet, B. Despax, and M. Caumont, J. of Phys. D: Appl. Phys. 35, 234239 (2002).

11 L. Cao, L. Laim, C. Ni, B. Nabet, and J. Spanier, J. of the Am. Chem. Soc. 127, 13782 (2005).

${ }^{12}$ Y. Miyamoto and M. Hirata, J. of the Phys. Soc. of Japan 44, 181 (1978).

13 F. J. Lopez, E. R. Hemesath, and L. J. Lauhon, Nano Lett. 9, 2774 (2009).

14 X. Liu and D. Wang, Nano Res. 2, 575 (2009).

15 E. Dickson and D. W. Pashley, Phil. Mag. 7, 1315 (1962). 
16 D. Pashley and M. Stowell, Phil. Mag. 8, 1605 (1963).

17 A. Carim, K. Lew, and J. Redwing, Advanced Mat. 13, 1489 (2001).

18 P. Buffat, M. Flüeli, R. Spycher, P. Stadelmann, and J. Borel, Faraday Discussions 92, 173 (1992).

19 C. Cayron, M. den Hertog, L. Latu-Romain, C. Mouchet, C. Secouard, J. Rouvière, E. Rouviere, and J. Simonato, Appl. Crystallography 42, 242 (2009).

${ }^{20}$ H. Kohno, N. Ozaki, H. Yoshida, K. Tanaka, and S. Takeda, Crystal Res. Tech. 38, 1082 (2003).

21 D. Cherns, Phil. Mag. 30, 549 (1974).

22 B. Korgel, D. Lee, T. Hanrath, M. Yacaman, A. Thesen, M. Matijevic, R. Kilaas, C. Kisielowski, and A. Diebold, IEEE Transactions on semiconductor manufacturing 19, 391 (2006).

23 J. Zhu, H. Peng, A. Marshall, D. Barnett, W. Nix, and Y. Cui, Nature Nanotech. 3, 477 (2008).

24 X. Dong-Yang, D. Lun, X. Wan-Jin, Y. Li-Ping, Z. Bo-Rui, R. Guang-Zhao, and Q. Guo-Gang, Chin. Phys. Lett. 23, 1317 (2006).

${ }^{25}$ F. Oehler, P. Gentile, T. Baron, M. den Hertog, J. Rouviére, and P. Ferret, Nano Tech. 20, 245602 (2009).

${ }^{26}$ F. Dhalluin, P. Desré, M. den Hertog, J. Rouvière, P. Ferret, P. Gentil, and T. Baron, J. of Appl. Phys. 102, 094906 (2007).

27 M. den Hertog, J. Rouviere, F. Dhalluin, P. Desré, P. Gentile, P. Ferret, F. Oehler, and T. Baron, Nano Lett. 8, 1544 (2008).

28 D. Buttard, T. David, P. Gentile, M. D. Hertog, T. Baron, P. Ferret, and J. Rouvière, Phys. Stat. Sol. A 250, 1606 (2008).

29 M. den Hertog, H. Schmid, D. Cooper, J. Rouviere, M. Björk, H. Riel, P. Rivallin, S. Karg, and W. Riess, Nano Lett. 9, 3837 (2009).

${ }^{30}$ K. Aissou, T. Baron, M. Kogelschatz, M. den Hertog, J. Rouvière, and J. Hartmann, Chem. Mat. 20, 6183 (2008).

31 D. Williams and C. Carter, Transmission Electron Microscopy (Springer, 1996).
32 J. Thibault, J. Rouvière, and A. Bourret, Materials Science and Technology, Volume 4, Electronic Structure and Properties of Semiconductors, chapter 7, Grain Boundaries in Semiconductors (Wiley-VCH, 1996).

33 H. Chen, Y. Gao, H. Yu, H. Zhang, L. Liu, Y. Shi, H. Tian, S. Xie, and J. Li, Micron 35, 469 (2004).

34 Z. Iqbal and S. Veprek, J. of Phys. C: Sol. State Phys. 15, 377 (1982).

35 Y. Zhang, Z. Iqbal, S. Vijayalakshmi, and H. Grebelb, Appl. Phys. Lett. 75, 2758 (1999).

36 J. Prades, J. Arbiol, A. Cirera, J. Morante, and A. F. i Morral, Appl. Phys. Lett. 91, 123107 (2007).

37 I. Zardo, S. Conesa-Boj, S. Estradé, L. Yu, F. Peiro, P. R. i Cabarrocas, J. Morante, J. Arbiol, and A. F. i Morral, Appl. Phys. A 100, 287 (2010).

38 B. Li, D. Yu, and S. Zhang, Phys. Rev. B 59, 1645 (1999).

39 S. Conesa-Boj, I. Zardo, S. Estradé, L. Wei, P. J. Alet, P. R. i Cabarrocas, J. Morante, F. Peiró, A. F. i Morral, and J. Arbiol, Crystal Growth and Design 10, 1534 (2010).

40 J. Einspahr and P. Voyles, Ultramicroscopy 106, 1041 (2006).

41 C. Kisielowski, B. Freitag, M. Bischoff, H. van Lin, Lazar, G. Knippels, P. Tiemeijer, M. van der Stam, S. von Harrach, M. Stekelenburg, et al., Microscopy and Microanalysis 14, 469 (2008).

42 H. Liu, S. Schmidt, H. Poulsen, A. Godfrey, Z. Liu, J. Sharon, and X. Huang, Science 332, 833 (2011).

43 V. Renard, M. Jublot, P. Gergaud, P. Cherns, D. Rouchon, A. Chabli, and V. Jousseaume, Nature Nanotech. 4, 654 (2009).

44 R. Algra, M. Hocevar, M. Verheijen, I. Zardo, G. Immink, W. van Enckevort, G. Abstreiter, L. Kouwenhoven, E. Vlieg, and E. Bakkers, Nano Lett. 11, 1690 (2011).

45 J. Arbiol, B. Kalache, P. R. i Cabarrocas, J. R. Morante, and A. F. i Morral, Nanotechnology 18, 305606 (2007).

46 P. Stadelmann, http: // cimewww. epfl. ch/people/ stadelmann/ jemsWebSite/ jems. html (2008), webpage. 